\title{
Quantitative light-induced \\ fluorescence technology for quantitative evaluation of tooth wear
}

\author{
Sang-Kyeom Kim \\ Hyung-Suk Lee \\ Seok-Woo Park \\ Eun-Song Lee \\ Elbert de Josselin de Jong \\ Hoi-In Jung \\ Baek-Il Kim
}




\title{
Quantitative light-induced fluorescence technology for quantitative evaluation of tooth wear
}

\author{
Sang-Kyeom Kim, ${ }^{\text {a }}$ Hyung-Suk Lee, ${ }^{a}$ Seok-Woo Park, ${ }^{a}$ Eun-Song Lee, ${ }^{a}$ Elbert de Josselin de Jong, ${ }^{\text {a,b,c }}$ \\ Hoi-In Jung, ${ }^{a, *}$ and Baek-II Kim ${ }^{a, *}$ \\ ${ }^{a}$ Yonsei University College of Dentistry, Oral Science Research Institute, Department of Preventive Dentistry and Public Oral Health, \\ BK21 PLUS Project, Seoul, Republic of Korea \\ bUniversity of Liverpool, School of Dentistry, Department of Health Services Research, Liverpool, United Kingdom \\ 'Inspektor Research Systems BV, Amsterdam, The Netherlands
}

\begin{abstract}
Various technologies used to objectively determine enamel thickness or dentin exposure have been suggested. However, most methods have clinical limitations. This study was conducted to confirm the potential of quantitative light-induced fluorescence (QLF) using autofluorescence intensity of occlusal surfaces of worn teeth according to enamel grinding depth in vitro. Sixteen permanent premolars were used. Each tooth was gradationally ground down at the occlusal surface in the apical direction. QLF-digital and swept-source optical coherence tomography images were acquired at each grinding depth (in steps of $100 \mu \mathrm{m}$ ). All QLF images were converted to 8-bit grayscale images to calculate the fluorescence intensity. The maximum brightness (MB) values of the same sound regions in grayscale images before $\left(\mathrm{MB}_{\text {baseline }}\right)$ and phased values after $\left(\mathrm{MB}_{\text {worn }}\right)$ the grinding process were calculated. Finally, 13 samples were evaluated. $\mathrm{MB}_{\text {worn }}$ increased over the grinding depth range with a strong correlation $(r=0.994, P<0.001)$. In conclusion, the fluorescence intensity of the teeth and grinding depth was strongly correlated in the QLF images. Therefore, QLF technology may be a useful noninvasive tool used to monitor the progression of tooth wear and to conveniently estimate enamel thickness. (C) 2017 Society of Photo-Optical Instrumentation Engineers (SPIE) [DOI: 10.1117/1.JBO.22.12.121701]
\end{abstract}

Keywords: tooth wear; occlusal wear; autofluorescence; quantitative light-induced fluorescence.

Paper 170119SSR received Feb. 22, 2017; accepted for publication Jun. 1, 2017; published online Oct. 17, 2017.

\section{Introduction}

Pathological tooth wear occurs progressively through the enamel and dentin layers by the interaction of mechanical and chemical factors. ${ }^{1-3}$ Tooth wear is an irreversible loss consisting of damage to the dental hard tissue. It can therefore affect the lifespan of the entire dentition. ${ }^{4}$ The progression of tooth wear to the stage of dentin exposure without preventive management or treatment requires restorative treatment. This is because the ensuing esthetic defect due to the loss of structural integrity or discoloration increases dentin hypersensitivity and the risk of pulpal exposure. Once the restorative treatment commences, it involves a prolonged and continuous course. In addition, the cost for the re-restoration and subsequent monitoring of the restored damage and natural teeth is considerable.,

Improvements in dietary or parafunctional habits and other etiological factors by continuous counseling and education for those at risk of developing pathological tooth wear or those who already have progressive teeth wearing are necessary to avoid or postpone the need for restorative treatment. Therefore, early diagnosis of tooth wear and monitoring of the process are crucial strategies. Finally, the goal of preventive interventions is to limit the loss of dental hard tissue to the enamel layer as much as possible. . $^{4-8}$

Indices based on subjective criteria using the naked eye and measurements of the decrease in length of the incisor clinical crown have been conventionally used to evaluate the severity

*Address all correspondence to: Baek-II Kim, E-mail: drkbi@yuhs.ac; Hoi-In Jung, E-mail: junghoiin@yuhs.ac of tooth wear. However, these subjective criteria are not particularly accurate or consistent because determining the presence of dentin exposure or the remaining enamel depends on highly subjective human decisions. ${ }^{9,10}$ Furthermore, although the latter strategy uses a relatively simple method to evaluate tooth wear, it also has limitations in its reflection of the wear of the palatal and posterior regions. ${ }^{7,11}$

Various technologies for the observation of tooth structure and the determination of decreased enamel thickness or wearinduced exposure of dentin have been suggested. These include profilometry, microradiography, scanning electron microscopy, and computer-aided design-computer-aided manufacturing laser scanning. ${ }^{8,12-14}$ However, most devices have clinical limitations. These limitations include difficulty of use in the oral cavity, considerable time requirements, and low resolution of images. ${ }^{7,13,15}$ Optical coherence tomography (OCT) is actively used in dentistry for the noninvasive observation of tooth structures. ${ }^{16}$ Because enamel and dentin have different scattering properties, OCT images can represent light-scattering intensity from different layers of dental hard tissue and provide information regarding enamel thickness. ${ }^{13}$ However, as the scanning range of OCT is only a few millimeters, it is not sufficient to screen large or multiple lesions in the dentition. In addition, image quality may be degraded due to insufficient processing time when rapid image acquisition is required. ${ }^{16}$

A previous study showed that teeth emit different fluorescence depending on the type and condition of the hard tissues, and particularly that of dentin, which emits a much more

$1083-3668 / 2017 / \$ 25.00$ (c) 2017 SPIE 


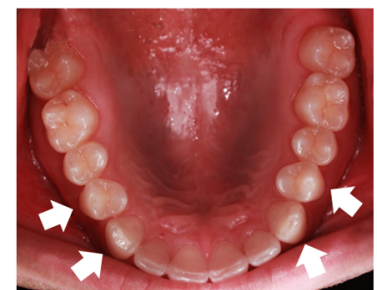

(a)

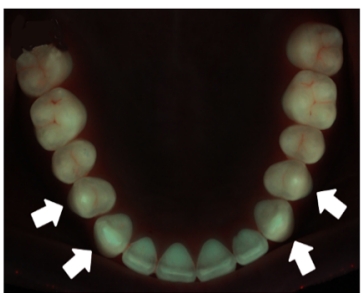

(b)

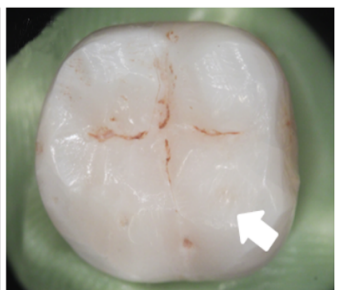

(c)

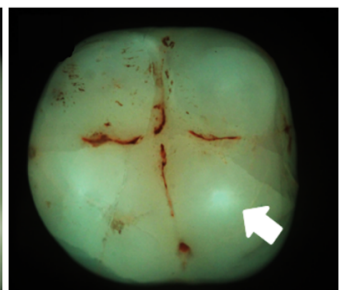

(d)

Fig. 1 QLF-D images of worn cusps showing bright fluorescence relative to other sites. (a) White-light and (b) fluorescence images of the occlusal surface of maxillary dentition. (c) White-light and (d) fluorescence images of extracted molars naturally worn in the oral cavity. All arrows represent wear facets indicating suspected dentin exposure or thinned enamel on cusps.

brilliant fluorescence signal than the enamel under ultraviolet excitation. ${ }^{17}$ Quantitative light-induced fluorescence (QLF) is an optical technology that detects the autofluorescence of a fluorophore, which is caused by excitation of fluorescent photons in the enamel and dentin layers following irradiation of the teeth with blue-violet light. ${ }^{18}$ Quantitative light-induced fluorescence digital (QLF-D) was introduced in dentistry as a QLF system using narrowband violet light $(405 \mathrm{~nm})$ and a high-specification digital single-lens reflex (DSLR) camera. ${ }^{19}$ Using photographs obtained with QLF-D, relatively bright fluorescence emission can be observed clinically in regions of suspected dentin exposure on occlusal surfaces of whole dentition (Fig. 1). QLF would thus be superior to OCT in terms of clinical convenience and efficiency. However, to the best of our knowledge, no current studies have reported differences in fluorescence between the enamel and the dentin in the evaluation of tooth wear. We thus aimed to determine the relationship between grinding depth and autofluorescence intensity of occlusal surfaces using QLF technology in vitro.

\section{Materials and Methods}

\subsection{Sample Collection and Serial Tooth Grinding}

This study was approved by the Institutional Review Board of Yonsei University Dental Hospital in the Republic of Korea (Approval No. 2-2015-0032). Sixteen permanent teeth (premolars) with no carious lesions or erosion on the occlusal surface were prepared as specimens. Artificial wear was induced by serial grinding of the occlusal surfaces. To minimize the distortion of the OCT images due to the surrounding tooth structure and to render the image analysis more convenient, premolars with well-developed buccal cusps compared with lingual cusps (mainly mandibular first premolars and maxillary first premolars with less-developed lingual cusps) were selected. The teeth were fixed to acryl molds using acrylic resin (Jet ${ }^{\mathrm{TM}}$ Tooth Shade; Lang Dental Manufacturing Co., Inc., Wheeling, Illinois). To confirm the grinding angle and to prevent excessive tooth wear, cylindrical acryl bars were attached next to the teeth.

A SS-OCT, prototype system (LG Electronics Inc., Seoul, Republic of Korea) was used to locate and select the measurement point on the cusp where the closest area of the enamel contoured to the dentinoenamel junction (DEJ) line. The distance from the measurement point to the base of the specimen was measured using digital calipers and was set to " 0 ." Subsequently, each specimen was ground using 400-grit grinding paper from the occlusal surface in the apical direction (Fig. 2). The grinding depth was serially calculated by
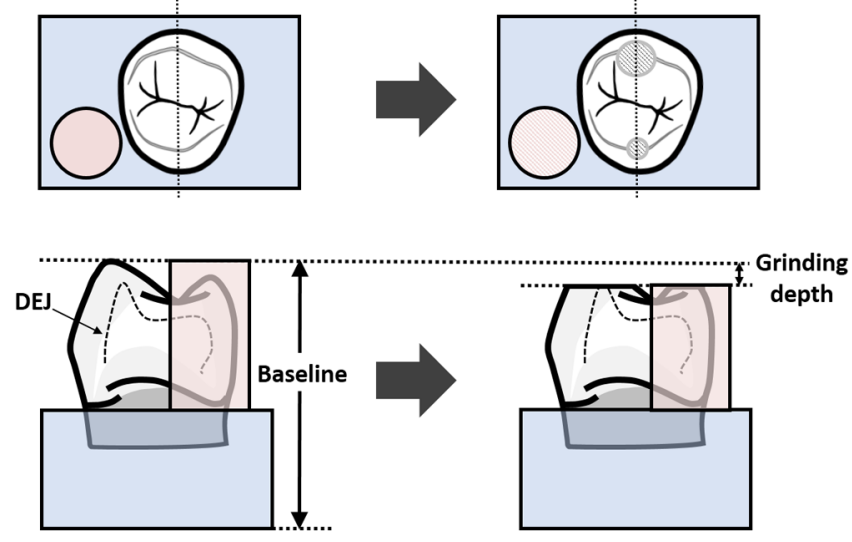

Fig. 2 Schematic diagrams of specimen and the grinding process.

measuring the length of the same measurement point. When the length reached " $-100 \mu \mathrm{m}$ " relative to the originally set " 0 " point, the QLF and OCT images were acquired, and the grinding was continued until the point of dentin exposure.

\subsection{Quantitative Light-Induced Fluorescence and Optical Coherence Tomography Image Acquisition}

At each measurement point, fluorescence images of the occlusal surface were acquired using a QLF-D Biluminator ${ }^{\mathrm{TM}} 2+$ system (Inspektor Research Systems BV, Amsterdam, The Netherlands). The specimens and QLF-D were fixed at the same location and angle each time the images were acquired. The cross-sectional (buccal-lingual) images of the measurement point that was selected at baseline were simultaneously acquired using OCT.

\subsection{Image Analysis}

The entire image analysis was conducted using the ImageJ software program version 1.50i (National Institutes of Health, Bethesda, Maryland). The fluorescence images were converted to 8-bit grayscale images so that we could calculate the fluorescence intensity by measuring brightness (gray value). ${ }^{20}$ The area of interest (AOI) was equally contoured and saved for each tooth. This area included the region with changes in fluorescence on the gray scale. To include all areas wherein changes in fluorescence intensity occurred on the worn surfaces, the AOIs were set based on the final images after completing the 
grinding procedure. The maximum brightness $(\mathrm{MB})$ values of each AOI were measured to determine changes in fluorescence intensity at each grinding depth. The MB of the AOI at baseline $\left(\mathrm{MB}_{\text {baseline }}\right)$ and that at each $100-\mu \mathrm{m}$ distance for each grinding depth $\left(\mathrm{MB}_{\text {worn }}\right)$ were measured, and the mean $\mathrm{MB}_{\text {baseline }}$ and each $\mathrm{MB}_{\text {worn }}$ value were calculated.

A retrospective analysis was conducted to determine changes in fluorescence over the remaining enamel thickness. After completing the grinding procedure, the dentin exposure points of all the teeth were set to " $0 \mu \mathrm{m}$," and the remaining enamel thickness and average MB values corresponding to each enamel thickness $\left(\mathrm{MB}_{\text {enamel }}\right)$ were calculated from $0 \mu \mathrm{m}$ (dentin exposure point) to $600 \mu \mathrm{m}$ at each $100 \mu \mathrm{m}$. The minimum grinding depth was $600 \mu \mathrm{m}$ for exposure of the dentin in all samples. Therefore, the calculated enamel thickness ranged from 0 to $600 \mu \mathrm{m}$ except for the higher range.

\subsection{Statistical Analysis}

All statistical analyses were conducted using the Statistical Package for the Social Sciences (SPSS) version 23.0 (SPSS Inc., Chicago, Illinois) with a significance level of 0.05 . The Pearson's correlation analysis was performed between the grinding depth and the average $\mathrm{MB}_{\text {worn }}$ values to determine the relationship between the degree of grinding and changes in fluorescence intensity. In addition, an independent $t$ test was performed between the average $\mathrm{MB}_{\text {baseline }}$ and each average $\mathrm{MB}_{\text {worn }}$ over the entire grinding depth to determine the point representing a significant difference in the fluorescence intensity due to the grinding.

A Pearson's correlation analysis was conducted between the remaining enamel thickness and the average $\mathrm{MB}_{\text {enamel }}$ values to determine changes in fluorescence emission based on enamel thickness. Furthermore, independent $t$ tests among all average $\mathrm{MB}_{\text {enamel }}$ values were conducted to determine differences in fluorescence intensity over the enamel thickness at $100-\mu \mathrm{m}$ intervals.

\section{Results}

Figure 3 shows the changes in the representative SS-OCT and QLF-D images and the MB values following the serial tooth grinding procedure. Thirteen samples were included in the final analysis of the results of this study. Three samples were excluded from the final analysis because the OCT detection of their DEJ contour was limited by crack lesions near the measurement points.

Each of the $\mathrm{MB}_{\text {worn }}$ values showed a tendency to increase over the grinding depth with a strong positive correlation (correlation coefficient $r=0.922$ to $0.997, P<0.001$ ). The average $\mathrm{MB}_{\text {worn }}$ values of all samples also showed strong correlations with grinding depth (correlation coefficient $r=0.994$, $P<0.001)$. There were significant differences between the average $\mathrm{MB}_{\text {baseline }}$ and all $\mathrm{MB}_{\text {worn }}$ values over the $200-\mu \mathrm{m}$ grinding depth $[P<0.05$, Fig. $4($ a) $]$.

Each of the $\mathrm{MB}_{\text {enamel }}$ values showed a tendency to decrease over the enamel thickness with a strong negative correlation (correlation coefficient $r=-0.870$ to $-0.992, P<0.05$ ). The remaining enamel thickness and the average $\mathrm{MB}_{\text {enamel }}$ also had a strong negative correlation $(r=-0.990, P<0.001)$. In addition, there were significant differences among the average $\mathrm{MB}_{\text {enamel }}$ values at $200-, 100-$, and $0-\mu \mathrm{m}$ enamel thickness $[P<0.01$, Fig. 4(b)]. The average rate of increase $(\%)$ of the $\mathrm{MB}_{\text {enamel }}$ was $4.00 \%$ for the range of enamel thickness from 600 to $200 \mu \mathrm{m}$, whereas this value was relatively higher in the range of enamel thickness from $200 \mu \mathrm{m}$ to the dentin exposure point $(6.51 \%)$.

\section{Discussion}

To the best of our knowledge, this is the first study to investigate the feasibility of using the difference in fluorescence intensity between the enamel and the dentin to evaluate tooth wear and estimate enamel thickness using QLF technology. As the grinding progressed, the fluorescence intensity was significantly increased by the simulated serial occlusal wear $(r=0.994$,

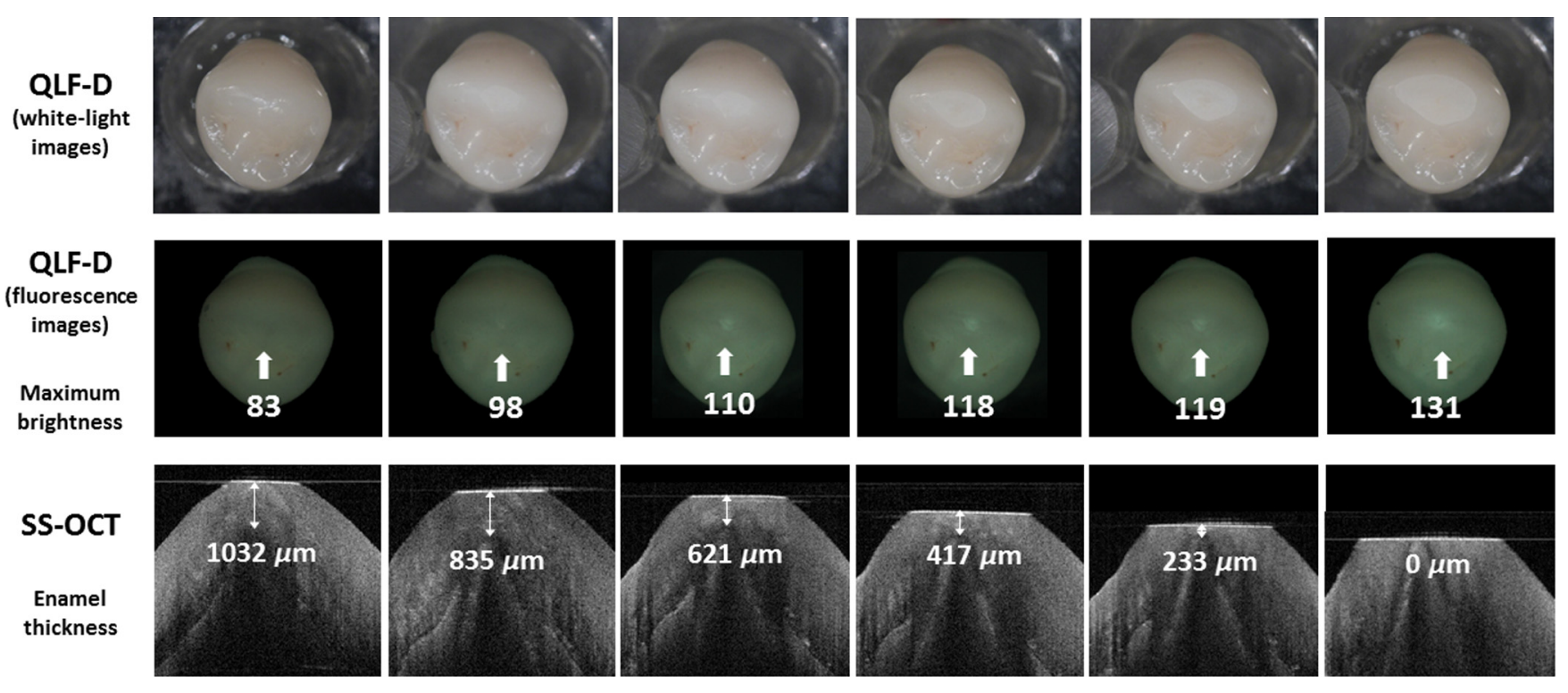

Fig. 3 Representative QLF-D images (white-light and fluorescence images) and SS-OCT of specimens in serial remaining enamel thickness. MB values were calculated from grayscale images converted from fluorescence images. The two-way arrows represent the enamel thickness (the nearest distance from the enamel surface to the DEJ line). 
(a)

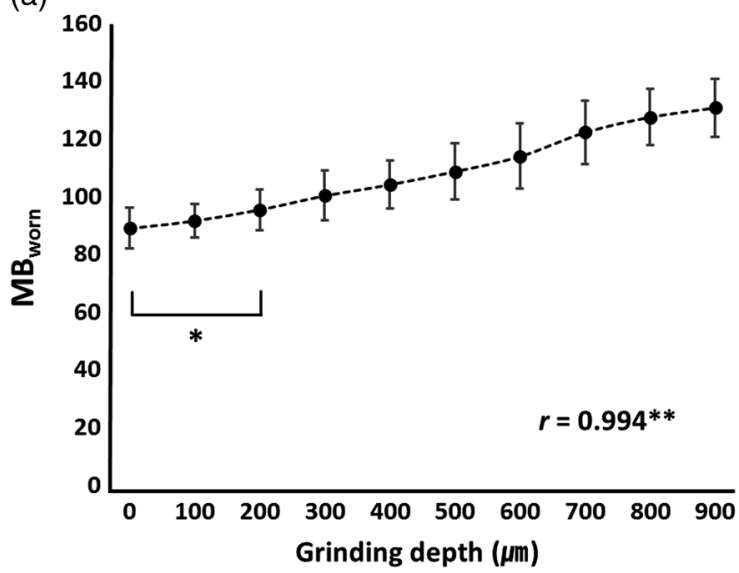

(b)

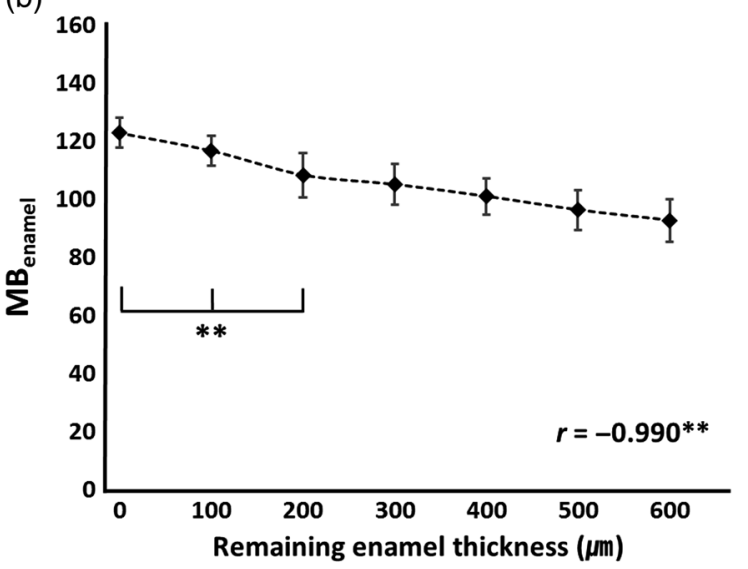

Fig. 4 Change in average $M B$ values with (a) increasing grinding depth $\left(\mathrm{MB}_{\text {worn }}\right)$ and (b) remaining enamel thickness $\left(\mathrm{MB}_{\text {enamel }}\right)$. The error bars represent standard deviations. (a) There were significant differences between the average $\mathrm{MB}_{\text {baseline }}$ and all $\mathrm{MB}_{\text {worn }}$ values over the $200-\mu \mathrm{m}$ grinding depth. (b) There were significant differences between the average $\mathrm{MB}_{\text {enamel }}$ values at 200-, 100-, and $0-\mu \mathrm{m}$ enamel thickness. $* P<0.05$ and $* * P<0.01$.

$P<0.001)$. A previous study using the QLF technique suggested that thick enamel has lower fluorescence intensity than thin enamel because of the longer distance through which the light is transmitted, which in turn increases light absorption and scatter in the enamel layer. This is a major piece of evidence that may describe the results obtained in this study. ${ }^{21}$

In contrast to images obtained using previous versions of the QLF system, the sound tooth surfaces have a whitish color in QLF-D images. ${ }^{22}$ Therefore, the conversion of the fluorescence images to grayscale images enables the measurement of fluorescence intensity as brightness values. The ability of QLF-D to distinguish between various resin composites and natural enamel surfaces due to the varying fluorescence intensities emitted by resin composites has already been reported. ${ }^{23}$ It has also been demonstrated that it is possible to detect subtle changes in fluorescence intensity on worn tooth surfaces using QLF-D.

Three samples were excluded from the analysis because their DEJ contours on OCT were limited due to crack lesions on or near the measurement points. However, it was possible to observe changes in fluorescence intensity during the grinding procedure in the QLF images. The effect of the natural morphology of occlusal surfaces on the transmission (penetration) of light has been reported as a limitation of OCT image analysis in studies investigating tooth structure or measuring enamel thickness using OCT. ${ }^{12,16,24}$ The crack lesions may also have affected the OCT images acquired in this study. These cracks appeared to be relatively dark in the QLF images likely due to a similar reason. ${ }^{25}$ Therefore, the worn regions can be clearly distinguished from cracks using the QLF technique. Furthermore, as the MB values were used in the image analysis, a more objective evaluation was possible by excluding errors related to anatomical factors or cracks that darken specific areas in the QLF images.

For the real-time observation of the serial changes in fluorescence intensity over the grinding process, we measured the grinding depth using calipers and confirmed our measurements using OCT images, which were used as the gold standard. This method substituted histological analysis in our study. The retrospective analysis was necessary to determine changes in fluorescence intensity based on the remaining enamel thickness instead of the grinding depth as the samples had various enamel thickness values at baseline. Therefore, each dentin exposure point was selected as a starting point, and the matched remaining enamel thickness at $100-\mu \mathrm{m}$ intervals was recalculated in the reverse order of each sample grinding depth. These results revealed that the enamel thickness and $\mathrm{MB}_{\text {enamel }}$ also had a strong correlation $(r=-0.990, P<0.001)$. The standard deviation of each $\mathrm{MB}_{\text {enamel }}$ was lower than that of the $\mathrm{MB}_{\text {worn }}$ and showed a tendency to gradually decrease as the enamel thickness approached the dentin exposure point. These results highlight the possibility of estimating the enamel thickness and determining dentin exposure using changes in fluorescence intensity.

Considering the importance of early diagnosis and monitoring of tooth wear, an objective method using sensitive instrumentation is necessary for the detection of the progression of tooth wear and dentin exposure. ${ }^{8}$ Although this was an in vitro study, the use of QLF in the detection of fluorescence changes induced by the grinding depth and decreasing enamel thickness, even at differences of $100 \mu \mathrm{m}$, would be clinically significant. Therefore, it is recommended that QLF be applied to the management of subjects who are expected to progress to pathological tooth wear by detecting subtle changes in fluorescence due to tooth wear and confirming speed of progression. Furthermore, the higher increase rate of fluorescence intensity from the $200-\mu \mathrm{m}$ enamel thickness to the dentin exposure point than that observed from 600 to $200 \mu \mathrm{m}$ was determined at similar intensities in comparable enamel thickness. Therefore, the present study results may serve as preliminary data for the diagnosis, monitoring, and intensive management of the early stages of enamel wearing.

QLF allows the noninvasive acquisition of fluorescence images of teeth in the oral cavity using harmless visible light. It will have advantages in confirming the tendency of tooth wearing and visualizing images in real time without the limitations of frequency and period of acquiring the relevant images. Considering these strengths, QLF may be a potential supportive diagnostic instrument for long-term preventive management, education, and longitudinal epidemiological investigation of tooth wear. Furthermore, interlocking the QLF-D technology 
with a DSLR camera enables the simple acquisition of high-resolution images of the entire dentition. Therefore, the time spent on detection, diagnosis, and performance of screening tests may be reduced when it is difficult to distinguish the exposed dentin using the naked eye.

Tooth wear is common in the cervical area as well as in the occlusal area due to the hard tissue loss in the cementoenamel junction. ${ }^{26}$ Cervical wear leads to lesions caused by the interaction of mechanical and chemical factors. ${ }^{27,28}$ In addition, when the occlusion is not ideal, weighted stress due to the occlusion can be a major factor contributing to cervical wear. ${ }^{29}$ Using the QLF technique, it would be possible to directly detect wear in the cervical region or to determine complex patterns of occlusal and cervical wear due to abnormal occlusion. QLF is also expected to provide visual information regarding the hypersensitivity caused by gingival recession and cervical dentin exposure.

Fluorescence image visualization and analysis using QLF had excellent stability in vitro. The experimental method used in this study detected subtle changes in the repetitive tooth wear process, suggesting that it has high reproducibility. However, to obtain reproducibility and stability of analysis using QLF in the clinical environment, the ambient light conditions should be kept as constant as possible when obtaining repetitive QLF images. Blocking of external light provides an ideal environment for increasing image quality. Additionally, relative analysis using the difference in standardized fluorescence intensity between sound enamel and exposed dentin through advanced analysis methods and algorithms will further improve the reproducibility and accuracy of the results. This will be possible using the QLF-dedicated analysis program under development.

Further studies would be necessary to confirm the direct relationship between the fluorescence intensities and the remaining enamel thickness of worn teeth in the oral cavity or a simulated environment. In addition, a demonstration that fluorescence emissions differ depending on the various causes and symptoms of tooth wear would be the ultimate goal. This technique may serve as the major indicator used for the differential diagnosis of tooth wear using fluorescence.

\section{Conclusions}

The autofluorescence intensity of the cusps was shown to gradually increase based on the progression of mechanical occlusal tooth wear, and both parameters showed a strong correlation. In particular, the fluorescence intensity increased rapidly over the $200-\mu \mathrm{m}$ remaining enamel thickness to the dentin exposure point.

\section{Disclosures}

Inspektor Research Systems BV provided the salary for author EdJdJ but did not have any additional role in the study design, data collection, analysis, decision to publish, or preparation of the paper. EdJdJ's involvement in this research was under the auspices of his status as adjunct professor at Yonsei University College of Dentistry supported by Brain Pool Program and BK21 PLUS Project. The specific role of EdJdJ was to provide his expertise regarding the fluorescence technology. This does not alter the author's adherence to the policies of the Journal of Biomedical Optics on sharing data and materials. EdJdJ holds several patents with respect to QLF technology. The remaining authors declared no conflicts of interest.

\section{Acknowledgments}

This study was supported by a grant from the Korean Federation of Science and Technology Societies (KOFST) funded by the Korean Government (Ministry of Science, ICT and Future Planning, MSIP), and Korea Institute of Planning and Evaluation for Technology in Food, Agriculture, Forestry and Fisheries (IPET) through High Value-added Food Technology Development Program, funded by Ministry of Agriculture, Food and Rural Affairs (MAFRA) (316071031HD020). Hoi-In Jung has contributed as a co-corresponding author with the corresponding author, Baek-Il Kim.

\section{References}

1. J. O. Grippo, M. Simring, and S. Schreiner, "Attrition, abrasion, corrosion and abfraction revisited: a new perspective on tooth surface lesions," J. Am. Dent. Assoc. 135(8), 1109-1118 quiz 1163-1105 (2004).

2. R. P. Shellis and M. Addy, "The interactions between attrition, abrasion and erosion in tooth wear," Monogr. Oral Sci. 25, 32-45 (2014).

3. A. Lussi, T. Jaeggi, and D. Zero, "The role of diet in the aetiology of dental erosion," Caries Res. 38(Suppl 1), 34-44 (2004).

4. A. Lussi and T. S. Carvalho, "Erosive tooth wear: a multifactorial condition of growing concern and increasing knowledge," Monogr. Oral Sci. 25, 1-15 (2014).

5. D. W. Bartlett, "Retrospective long term monitoring of tooth wear using study models," Br. Dent. J. 194(4), 211-213 (2003).

6. P. Lambrechts et al., "Restorative therapy for erosive lesions," Eur. J. Oral Sci. 104(2), 229-240 (1996).

7. P. Wetselaar and F. Lobbezoo, "The tooth wear evaluation system: a modular clinical guideline for the diagnosis and management planning of worn dentitions," J. Oral Rehabil. 43(1), 69-80 (2016).

8. R. G. Chadwick et al., "Development of a novel system for assessing tooth and restoration wear," J. Dent. 25(1), 41-47 (1997).

9. C. Ganss, J. Klimek, and A. Lussi, "Accuracy and consistency of the visual diagnosis of exposed dentine on worn occlusal/incisal surfaces," Caries Res. 40(3), 208-212 (2006).

10. V. Margaritis and J. Nunn, "Challenges in assessing erosive tooth wear," Monogr. Oral Sci. 25, 46-54 (2014).

11. P. Magne, G. O. Gallucci, and U. C. Belser, "Anatomic crown width/ length ratios of unworn and worn maxillary teeth in white subjects," J. Prosthet. Dent. 89(5), 453-461 (2003).

12. A. Algarni et al., "Enamel thickness determination by optical coherence tomography: in vitro validation," Caries Res. 50(4), 400-406 (2016).

13. T. Attin and F. J. Wegehaupt, "Methods for assessment of dental erosion," Monogr. Oral Sci. 25, 123-142 (2014).

14. M. K. Al-Omiri et al., "Quantification of incisal tooth wear in upper anterior teeth: conventional vs. new method using toolmakers microscope and a three-dimensional measuring technique," J. Dent. 41(12), 1214-1221 (2013).

15. R. DeLong, "Intra-oral restorative materials wear: rethinking the current approaches: how to measure wear," Dent. Mater. 22(8), 702-711 (2006).

16. Y.-S. Hsieh et al., "Dental optical coherence tomography," Sensors 13(7), 8928-8949 (2013).

17. H. C. Benedict, "A note on the fluorescence of teeth in ultra-violet rays," Science 67(1739), 442-442 (1928).

18. M. H. van der Veen and E. de Josselin de Jong, "Application of quantitative light-induced fluorescence for assessing early caries lesions," Monogr. Oral Sci. 17, 144-162 (2000).

19. C. K. Hope et al., "Photobleaching of red fluorescence in oral biofilms," J. Periodontal Res. 46(2), 228-234 (2011).

20. R. S. A. A. D. Reis et al., "Evaluation of fluorescence of dental composites using contrast ratios to adjacent tooth structure: a pilot study," J. Esthet. Restor. Dent. 19(4), 199-206 (2007).

21. M. Ando et al., "Influence of enamel thickness on quantification of mineral loss in enamel using laser-induced fluorescence," Caries Res. 37(1), 24-28 (2003).

22. G. Felix Gomez, G. J. Eckert, and A. Ferreira Zandona, "Orange/red fluorescence of active caries by retrospective quantitative light-induced fluorescence image analysis," Caries Res. 50(3), 295-302 (2016). 
23. B. R. Kim et al., "Differences in the intensity of light-induced fluorescence emitted by resin composites," Photodiagn. Photodyn. Ther. 13, 114-119 (2016).

24. R. S. Jones et al., "Imaging artificial caries on the occlusal surfaces with polarization-sensitive optical coherence tomography," Caries Res. 40(2), 81-89 (2006).

25. M. K. Jun et al., "Detection and analysis of enamel cracks by quantitative light-induced fluorescence technology," J. Endod. 42(3), 500-504 (2016).

26. L. C. Levitch et al., "Non-carious cervical lesions," J. Dent. 22(4), 195-207 (1994).

27. J. D. Bader et al., "Case-control study of non-carious cervical lesions," Community Dent. Oral Epidemiol. 24(4), 286-291 (1996).
28. A. Lussi and M. Schaffner, "Progression of and risk factors for dental erosion and wedge-shaped defects over a 6-year period," Caries Res. 34(2), 182-187 (2000).

29. W. C. Lee and W. S. Eakle, "Possible role of tensile stress in the etiology of cervical erosive lesions of teeth," J. Prosthet. Dent. 52(3), 374-380 (1984).

Sang-Kyeom Kim is a PhD candidate in the Department of Preventive Dentistry and Public Oral Health at Yonsei University. He received a BS degree in dental hygiene from Namseoul University in 2015. His current research interests include early detection of oral disease and clinical application of fluorescence-based devices.

Biographies for the other authors are not available. 